\title{
AGENT TECHNOLOGY FOR VIRTUAL ORGANIZATIONS
}

\author{
Vladimír Mařík \\ Department of Cybernetics, Czech Technical University in Prague, Czech Republic \& \\ Rockwell Automation Research Center, Americka 22, 12000 Prague, CZECH REPUBLIC, \\ marik@labe.felk.cvut.cz \\ Michal Pěchouček \\ Department of Cybernetics, Czech Technical University in Prague, CZECH REPUBLIC \\ pechouc@labe.felk.cvut.cz
}

\begin{abstract}
The paper summarizes the latest achievements in the area of intelligent agent-based solutions for manufacturing. The main idea of this paper is that many solutions developed in the multi-agent area can be successfully reused for the virtual enterprise purposes. Critical analysis of both the MAS advantages and gaps from the VO point of view is presented.
\end{abstract}

\section{INTRODUCTION}

Both the complexity of the environment where we live and operate as well as the complexity of tasks to be solved is growing rapidly. In many situations, the centralized and hierarchically organized decision-making and solutions are not adequate and fail just because of complexity of tasks to be solved and requirements to be put on these tasks.

To overcome the complexity barrier, it is quite natural to consider sets of autonomous, efficiently cooperating units being easily integrated by the "plug-andplay" approach instead of the rigid, centralized architectures. This is a clearly visible trend on all the levels of manufacturing and businesses. On the real-time level where these units are tightly physically linked to the manufacturing hardware we talk about holons and holonic solutions; intelligent agents are helping to solve the production planning and scheduling tasks on the workshop and plant levels. The vision of internet-based cooperation among enterprises has lead to the idea of virtual enterprises: "A virtual enterprise is a temporary alliance of enterprises that come together to share skills or core competencies and resources in order to better respond to business opportunities, and whose cooperation is supported by computer networks." (Camarinha-Matos \& Afsarmanesh, 1999).

The philosophical background of all these approaches is the same: efficient coordination and cooperation among autonomous intelligent goal-oriented units can lead to a quite effective behavior of the community as a whole. Knowledge stored and applied locally provides interesting and useful patterns of emergent behavior. 
The area of virtual enterprises is usually widely discussed from many diverse aspects (like e.g. social, ethical, legal, technical, technological, etc.), but it lacks in more formal specifications, models, architectures, scenarios, and standards (Camarinha-Matos \& Afsarmanesh, 2002). On the other hand, such formalisms are already - in this or other form - available and successfully applied in the area of multi-agent systems. Why not to overtake these for the purposes of virtual enterprises and organizations? The same is true for the supporting tools and platforms. And they have many features in common: e.g. each agent/unit represents one functional entity, knowledge of all kind is stored locally and explored intensively, the operation space in which the unit operates provides enough freedom for units to appear, disappear and be modified easily etc.

The theory and technology of multi-agent systems can enrich the formalisms and tools to be applied in the VO field. Let's talk about several specific techniques from the multi-agent area, which would be immediately applicable in the area of VOs. These are e.g.

- An agent architecture usually consists of the agent's body and the agent's wrapper (Maŕík et al., 2001). The wrapper accounts for the inter-agent communication and real-time reactivity. The body is an agent's reasoning component. It very often happens that the agent's body is a pre-existing piece of software that is integrated by the agent's wrapper into the agent structure. From the outside such a software system cannot be distinguished from an "ordinary" agent. This agentification activity provides an elegant mechanism for system integration.

- acquaintance models used located in the wrappers of individual agents are used to organize, maintain and explore knowledge about the other agents (about their addresses, capabilities, load, reliability etc.). This kind of knowledge, which strongly supports collaboration activities among the agents, is called social knowledge (Maŕík et al., 2001).

- techniques for organizing alliances and coalitions and planning of their activities (team action planning) (Tambe, 1997).

- techniques for administration and maintenance of private, semi-private and public knowledge. This seems to be an important issue to tackle e.g. especially in VEs of temporary nature where units competing in one business project are also contracted to cooperate in the other one.

- meta-agent technology can be explored to monitor the activities of the partners in the VE from a higher-level perspective, to reason about this activity and to infer new rules for the VE community operation. Using this approach, the regulative and supportive roles of e.g. Chambers of commerce, tax authorities or other legislative bodies can be represented/simulated.

- standardized negotiation techniques and protocols are available in the multiagent area. These are based e.g. on contract-net-protocol, or various versions of auctions, on acquaintance models based negotiations (e.g. the subscribe-advertise technique). If at least part of the negotiation processes is based on the acquaintance model, an important feature for VEs can be achieved: the optimality of contracting from the long-term point of view (not just the optimality for one business case).

- standardization and interoperability principles can be simply transferred into the VO area. The same or similar interoperability rules applied on all the manufacturing and business management levels (starting at holonic real-time control up to VOs) would even help to integrate these levels directly later. Thus, the 
planning department of one VO partner would be enabled to "talk" directly with the real-time control level of the other partner if needed.

- security and authethication is a vital and important field in the agent relatedresearch (Novák et al., 2003).

For better understanding of the technologies and formalisms available in the field of multi-agent systems, let's present a set the formalisms developed and successfully used. Formalisms, which can be directly applied to solve crucial tasks of knowledge handling and exploration in VOs, have been preferably chosen as a source of inspiration. Later on in this paper, we will also discuss possible contribution of the MAS technologies to the area of VOs.

\section{AGENT'S NEIGHBORHOOD}

Each agent may participate in one group of 'friendly' agents (alliance) and at the same time it may be actively involved in several groups (coalitions) of agents cooperating in fulfilling specific shared tasks (Shehory \& Krause, 1998), (Pěchouček et al., 2002). Computational and communication complexity of forming such a group (coalition) depends on the amount of pre-prepared information the agents administer one about the other and on sophistication of the agents' capability to reason about the other agents' resources, plans and intentions. The agents can allow others to reason about them and at the same time they can reason differently about the agents that belong to their different scopes of reasoning - neighborhood. Therefore, we distinguish among several types of agents' neighborhoods:

$-\alpha(A)$ - agent's total neighborhood, a set of all agents that the agent $A$ is aware of, (e.g. knows about their existence and is able to communicate with them)

$-\mu(A)$ - agent's social (monitoring) neighborhood that is a set of agents, which the agent $A$ keeps specific information about (e.g. services they provide, status, load, etc.). This neighborhood consists of the set of the agents that the agent $A$ reasons about $-\mu^{+}(A)$ and the set the agents that reason about the agent $A-\mu^{-}$ (A). Therefore $\forall B \in \mu^{-}(A): A \in \mu^{+}(B)$.

$-\varepsilon(A)$ - agent's cooperation neighborhood that is a set of agents jointly collaborating (or committed to do so) in achieving one or more shared goals.

\section{ALLIANCE, COALITION, TEAM ACTION PLAN}

We will understand as the multi-agent community $\Theta$ the whole collection of agents participating in a business task. We will introduce the concept of an alliance as a collection of agents that share information about their resources and all agree to form possible coalitions (Pěchouček et al., 2002). The alliance is regarded as a longterm cooperation agreement among the agents. Members of an alliance will all belong to one others' social neighborhood. Provided that we assume that each agent belongs also to its own social neighborhood $-\forall A \in \Theta: A \in \mu(A)$, we define the alliance as follows: 
An alliance is a set of agents $\kappa$, so that $\forall A \in \Theta: \exists \kappa: A \in \kappa$ $\wedge \forall A_{\mathrm{i}} \in \kappa: \kappa=\mu\left(A_{\mathrm{i}}\right)$.

A singleton agent is regarded as an alliance with just one member. From the requirements for the reciprocal knowledge sharing within an alliance follows that

$$
\forall A \in \mathrm{K}: \mathrm{\kappa}=\mu(A) \text {. }
$$

Therefore, an important property of an alliance is that it cannot overlap with another alliance:

$$
\forall \kappa_{1}, \kappa_{2} \subseteq \Theta:\left(\exists A: A \in \kappa_{1} \wedge A \in \kappa_{2}\right) \Rightarrow \kappa_{1} \equiv \kappa_{2} .
$$

Let us define a coalition as a set of agents, which agreed to fulfill a single, wellspecified goal. Coalition members committed themselves to collaborate on the within-coalition-shared goal. Under the assumption $\forall A \in \Theta: A \in \varepsilon(A)$ we define coalition as follows:

$$
\begin{aligned}
& \text { A coalition is a set of agents } \chi \text {, so that } \forall \chi(\tau) \subseteq \Theta: \forall A \in \\
& \chi(\tau): \chi(\tau) \subseteq \varepsilon(\mathrm{A}) \text {. }
\end{aligned}
$$

Let us introduce a set $\varepsilon(A, \tau)$ that is an agent collaboration neighborhood with respect to a single shared goal $\tau$. Then

$$
\varepsilon(A)=\quad \varepsilon(A, \tau) \text {, and } \forall \chi_{\tau}(\tau) \subseteq \Theta: \forall A \in \chi(\tau): \chi(\tau)=\varepsilon(A, \tau) .
$$

A coalition, unlike an alliance, is usually regarded as a short-term agreement between collaborative agents. As we will see in Section 6, it is better for a coalition to be a subset of one alliance, but it is not an inevitable condition. A coalition can consist of agents who are members of different alliances.

Another term that we have to introduce is a team action plan. In planning any task, the agents must agree on how they will achieve the goal $\tau$. The team action plan is thus a decomposition of a goal $\tau$ into a set of tasks $\left\{\tau_{i}\right\}$. The tasks will be delegated within the coalition members. Apart from the responsible agent, each task shall be denoted by its due time, start time and price. Provided that an agent $A_{\mathrm{j}}$ is responsible for implementing the task $\tau_{\mathrm{i}}$ (where $\tau=\left\{\tau_{\mathrm{i}}\right\}$ ) in time due $\left(\tau_{\mathrm{i}}\right.$ ), starting at $\operatorname{start}\left(\tau_{\mathrm{i}}\right)$ for the price price $\left(\tau_{\mathfrak{i}}\right)$, we define the team action plan as follows:

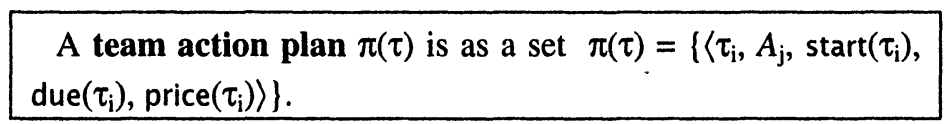

We say that the team action plan $\pi(\tau)$ is correct if all the collaborators $A_{\mathrm{j}}$ are able to implement the task $\tau_{\mathrm{i}}$ in the given time and for the given price. The team action plan $\pi(\tau)$ is accepted if all agents $A_{\mathrm{j}}$ get committed to implementing the task $\tau_{\mathrm{i}}$ in the given time and for the given price. Similarly, we say about the goal $\tau$ to be achievable, if there exists such $\pi(\tau)$ that is correct. The goal $\tau$ is said to be planned, if there exists $\pi(\tau)$ that is accepted. Obviously, there is an important relation between the team action plan and the coalition. We say that a coalition $\chi(\tau)$ achieves a goal $\tau$ by implementing a team action plan $\pi(\tau)$ if and only if $\chi(\tau)=\left\{A_{j}\right\}$ and $\pi(\tau)$ is correct. 


\section{KNOWLEDGE SHARING \& KNOWLEDGE DISCLOSURE}

In order to reason one about the other, the agents must share some of their knowledge. Let us introduce the operator $(\mathrm{Bel} A \varphi)$ that expresses the agent's $A$ awareness of the formula $\varphi$ being true (Wooldridge 2000). We say that the agent $A_{0}$ intentionally shares its knowledge $K\left(A_{0}\right)$ with a set of agents $\delta\left(A_{0}\right) \subseteq \Theta$ provided that:

$$
\begin{gathered}
\mathrm{K}\left(A_{0}\right)=\{\varphi\}: \forall \varphi \in \mathrm{K}\left(A_{0}\right): \forall A_{\mathrm{i}} \in \delta\left(A_{0}\right):\left(\operatorname{Bel} A_{\mathrm{i}} \varphi\right)^{\wedge} \forall B_{\mathrm{i}} \notin\left\{\delta\left(A_{0}\right) \cup\left\{A_{0}\right\}\right\}:\left(\operatorname{Bel} A_{0}\right. \\
\left.\neg\left(\operatorname{Bel} B_{\mathrm{i}} \varphi\right)\right) .
\end{gathered}
$$

From the previous follows, that if an agent $B$ knows some of the shared information without the agent $A_{0}$ being aware of this fact, the agent $B$ is not regarded as a member of the $\delta\left(A_{0}\right)$ set of agents, representing $A_{0}$ 's knowledge sharing neighborhood. According to this classification, we suggest three levels of the agent's knowledge sharing:

Public Knowledge is shared within the entire multi-agent community. If it is assumed that all the agents know one about the other (i.e. $\forall A, A \in \Theta: \alpha(A)=\Theta$ ), public knowledge $\mathrm{K}_{\mathrm{P}}\left(A_{0}\right)$ of an agent $A_{0}$ is defined as

$$
\operatorname{Kp}\left(A_{0}\right)=\mathrm{K}\left(A_{0}\right) \text { where } \delta\left(A_{0}\right)=\alpha\left(A_{0}\right) .
$$

This class of knowledge is freely accessible within the community. As public knowledge we understand the agent's name, the type of the unit (workshop, plant, customer) the agent represents, the general objectives of the agent's activity, the country where the agent is registered, agent's human-human contact (telephone, fax number, email), the human-agent type of contact (http address), the agent-agent type of contact (the IP address, incoming port, ACL) and, finally, available services.

Semi-Private Knowledge is shared within agents' social neighborhoods. Semiprivate knowledge $\mathrm{K}_{\mathrm{s}}\left(A_{0}\right)$ of an agent $A_{0}$ is defined as

$$
\mathrm{Ks}\left(A_{0}\right)=\mathrm{K}\left(A_{0}\right) \text { where } \delta\left(A_{0}\right)=\mu\left(A_{0}\right) \text {. }
$$

As in the OOTW domain, we do not assume the knowledge to be shared within the overlapping alliances, we will require the social neighborhood to have the following property: $\forall A \in \Theta: \mu^{-}(A)=\mu^{+}(A)=\mu(A)$. Members of a social neighborhood share information about availability of their resources.

Private Knowledge is owned and administered by the agent itself. Private knowledge $\mathrm{Kp}\left(A_{0}\right)$ of an agent $A_{0}$ is defined as

$$
\operatorname{Kpr}\left(A_{0}\right)=\mathrm{K}\left(A_{0}\right) \text { where } \delta\left(A_{0}\right)=\{\},
$$

An important type of private knowledge includes agent's collaboration preferences, alliance restrictions, coalition leader restrictions and possible next restrictions, but also agent's planning and scheduling algorithms.

Measuring the loss of information, that the agents may want to keep private, is an uneasy task. The revealed piece of information has got different value to agents with different meta-reasoning capabilities (Pěchouček \& Norrie, 2000). In order to vaguely categorize various types of information leaks, let us distinguish between 
-Strong information disclosure: If an agent looses some type of private (or semi-private) knowledge in the strong sense, it does so as a side effect of some proactive step (such as sending a request).

- Weak information disclosure: If an agent looses the private knowledge in the weak sense, it deliberately discloses some piece of its knowledge to other agents (e.g. when sending an inform-type message).

Each agent undertakes the weak loss of some of its knowledge when forming an alliance. At this moment the agent's semi-private knowledge gets disclosed within the alliance members. The agent looses some of its private knowledge in the strong sense, if it communicates with an agent which is outside of its alliance. Once the agent $A_{1}$ from an alliance $\kappa_{1}$ sends a request for a service $\tau$ to the agent $A_{2}$ from the alliance $\kappa_{2}$, the agent $A_{1}$ reveals the information about the intent (e.g. $A_{1}$ does something that requires $\tau$ ) and information about agent's $A_{1}$ capabilities (e.g. $A_{1}$ cannot do $\tau$ ). At the same time, a proposal for collaboration from $A_{2}$ reveals the information about agent's $A_{2}$ capabilities (such as $A_{2}$ can do $\tau$ in time $t_{1}$ ). However, this type of knowledge disclosure has been reduced as $A_{2}$ acts on behalf of the entire alliance. Therefore, if $A_{2}$ offers some services that are not used at the end, there is a loss of information about capabilities of the entire alliance (and not of $A_{2}$ itself).

\section{ANALOGY BETWEEN MULTI-AGENT SYSTEMS AND VIRTUAL ENTERPRISES}

Let's consider each company in a VE is operating autonomously, carrying out its relevant duties locally, communicating and exchanging information with the others just when needed. Such a company, as a rule, wants to have the right to join and leave the VE freely, but it understands, that it should satisfy generally valid rules of behavior. In an ideal case, the VE is able to operate in a turbulent environment, to react on unpredictable situations by e.g. automatic re-configuration or extension/reduction of its capabilities and resources.

Let's try to formulate the above-mentioned analogy in more detail, discussing potential role of equivalent elements or functionalities developed or simulated in the area of MAS on one hand and expected to be achieved in the field of VEs on the other hand:

1. Each company can be considered as an autonomous unit, as an agent able to carry out specific (usually very complex) functions.

2. Each company entering the VE should register with the others, the "yellow pages" and "white pages" services are provided either in a centralized (if there is a strong VE leader) or decentralized way (in a flat, temporary networks).

3. Each member of the VE network is usually informed - at least in the extend needed for participation in the network - about the capabilities and resources of the others. This knowledge is usually concentrated in the business planning and scheduling units, which play the role of acquaintance models in the MAS terminology. Let's stress that the "social" knowledge contained in the acquaintance models is extremely important for efficiency of the VE as a whole.

4. Standardized way of knowledge and data exchange among the individual companies in the VE should be ensured by an interoperable IT infrastructure. 
The IT departments of the companies responsible for maintaining this infrastructure as well as for the correct format of messages exchanged, play the role of the communication part of the wrapper in the MAS terminology.

5. The processes of the VE formation as well as the joint planning and scheduling activities are based on jointly known - at least within the agents willing to create the VE - negotiation rules and scenarios. These are very similar (or identical) to those called protocols (like e.g. CNP) or auctions (e.g. Dutch or sealed) in the MAS domain.

6. The highly specialized members of a virtual enterprise, like brokers or professional network organizers, can find easily their counterparts in the MAS community, e.g. various middle agents and brokers (Sycara, 2001). The negotiation and brokering algorithms proved in the MAS area can be used to formalize (and to automate) the corresponding processes in virtual enterprises.

7. The entities which observe the activity of the network and which can influence the rules of operation or policies set in the network (like e.g. chambers of commerce, regional authorities, tax office, or new types of "normative institutions") can be represented by the meta-agents (either passive or active). The meta-agents could be also introduced by the virtual enterprises themselves as tools helping to detect the less efficient parts or bottlenecks of the network as well as providing advice supporting the self-evolution of the virtual enterprise in the desired direction. They are - as a rule - able to learn from their own experience. The other function can be offered by the meta-agents, namely the self-reflectivity, which can be used by the VE members to understand better the processes appearing within the frame of a VE from the global point of view.

8. The processes of the VE creation are analogous to coalition formation processes in the MAS domain: Usually there is a group of companies willing to cooperate sharing necessarily a certain part of the company confidential knowledge (this group might be labeled as an alliance and the shared knowledge as a semi-private in the MAS terminology). The alliance members negotiate the opportunity to create a VE for a given task (in MAS terminology: to create a coalition), there is - as a rule - one company overtaking the role of a VE leader (the coalition leader in MAS terminology). Once the VE is set, it is necessary to develop a joint production plan and, later, schedule (team action planning).

9. Virtual enterprises could use the mobile agents as well, e.g. for market research or for evaluation of potential members of the alliance. The mobile agents would move, in this case, to the individual customer's or partner's server to evaluate the relevant data and to inform the alliance/coalition partners about the results of evaluation. They die or turn their status to "a sleep" afterwards.

10. The specific security principles used in the MAS area can be re-used in the virtual enterprise domain as well.

\section{ROLE OF AGENT-BASED SIMULATION}

When considering the analogy principles mentioned above, it is clear that the agent technology can be explored in designing simulations tools and applications for the 
VEs with advantage. It is necessary just to decide which entities will be represented as a single agent, to define the specialized functionalities/units (like meta-agents, brokers etc.) and the corresponding bodies, which will represent them in the MAS system. The agent-based simulation could be used e.g.

- to predict the behavior of the $V E$ as a whole. The fact that there is no central unit in a VE or MAS represents a critical barrier in a wider applicability of both the MAS and VE ideas. The economists, designers and end-users are simply afraid of loosing control over the emergent behavior, which might occur in the community/network without any central element. They are afraid of stability and convergence of such an behavior. Simulation cansignificantly help to understand the internal processes in the community.

- to predict and test the optimal scenario for the VE development, to understand better, which type of the VE satisfy in the best way the requirements of the market or needs for the problem at hand,

- to select the most optimal negotiation strategy for each of the partners,

- to link directly the simulation with real emergent processes in a VO environment enables to use the simulation tools repeatedly in the real-time activities. This capability means that whereas part of the VO is engaged fully in the real-life activities, the remaining part can be just simulated. The shift of the borderline between the simulated and real part of the VE can be carried out in a quite smooth way.

\section{THE SAME PHILOSOPHY USED ACROSS VE}

There is the other important issue connected with the MAS approach when used in a virtual manufacturing enterprise. The same agent-oriented philosophy can be used on different levels:

- on the lowest level of real-time holonic control; this level is tightly linked with the physical manufacturing devices (Deen, 2003). The holons create holarchies and are sometimes wrapped into higher-level holonic or software agents, which are responsible for higher level negotiations needed in the case of a failure or when changes in the manufacturing plan suddenly occur,

- on the shop-floor and company decision-making level, the agent-based planning and scheduling may be introduced. Being partially extended to an extraenterprise planning and scheduling (when the internal shop-floor planning is permitted to be directly influenced or carried out by entities outside the company, e.g. by preferred customers of suppliers), an important technological step based on agent-based approach towards the virtual enterprise can be done.

- on the level of the virtual inter-company cooperation when appropriate IT platform, standard agent services, automated negotiation scenarios etc. are used to plan the coordination, cooperation and global functional integration.

When the same philosophy, stemming from the agent-oriented approach is used across the manufacturing and business process from the individual machines up to the virtual enterprise as a whole (in the horizontal direction), new interesting potential interactions do appear: E.g. the holonic agents from different companies can talk to each other asking for changes of some manufacturing parameters, the business department of one company can send data directly to the scheduling 
mechanism of a specific workshop belonging to a partner's company without the need to inform the managerial level, the manufacturing holons can directly warn the purchase unit avoiding classical hierarchical communication channels in the plant etc. To enable this across the company, peer-to-peer messaging and interactions requires the development of appropriate message portfolio and of corresponding knowledge containers (acquaintance models) placed in individual agents.

\section{MAS TECHNIQUES NOT ADEQUATELY DEVELOPED FOR THE VE NEEDS}

It is also possible to detect the topics, which are not solved by the MAS technologies in the extend needed for the virtual enterprises yet, e.g.

- The knowledge seems to be much more wider and diversified in the case of VEs. The adequate knowledge capture and management methods needed in virtual enterprises, where the extent of the knowledge exceeds the knowledge usually handled by the "classical" MAS systems by several orders, are still missing. The knowledge management approaches in the current MAS systems are just toy examples from the point of view of VEs. It is necessary to develop standardized, quite new techniques for capturing the vast volumes of knowledge, maintain it up-to-date and explore it in an efficient way.

- The current experience concerning the knowledge ontology representation, maintenance and exploration in MAS just helps to understand how important the common shared ontologies will be for the virtual enterprises and how important will be the mutual transformation among different ontologies. Simply, the ontologies in the MAS area are not developed enough to provide a direct support to the VE solutions.

- FIPA standardization efforts demonstrate one possible way, which the VE area should go to achieve the full interoperability. The FIPA standards developed and approved are not ready to support the interoperability in the area of VEs. But, a very intensive cooperation with the FIPA organization might speed-up the process of introducing interoperability principles in the area of VEs, either within or outside the frame of FIPA consortium.

- There are already several MAS platforms available, majority of them are FIPAcompliant (JADE, FIPA-OS, JACK etc.). Unfortunately, none of them is directly applicable in the field of VEs because of several reasons: The message structures and ontologies are not rich enough, they are underdeveloped from the point of view of VEs' needs. It is even not clear whether the current FIPA standards can be extended and modified in a way, which would be acceptable and reasonable for the VE area. But, in any case, FIPA results provide good inspiration and outline the initial pathway for the VE platform research.

\section{CONCLUSIONS}

The results achieved in the MAS area provide an excellent motivation for the development of solutions for virtual enterprises, which would be based on similar principles and technologies. Some principles and techniques can be re-used 
immediately, some of them are still underdeveloped and their current state of development just encompasses the way where to go.

The VE community lacks namely in an efficient IT platform, specially developed for the area of virtual enterprises. The general IT technology is mature enough to enable to develop such a platform, but the research, development and namely the investments into this platform should be done by one of the major IT players. This is the only chance how to get an adequate, high performance, and thus applicable, platform soon. Problems of developing such a platform seem to be

- the ability to manage exploration of vast volumes of highly distributed knowledge (some parts of it being considered as private or semi-private),

- interoperability of the communication interfaces which would enable rich communication, which would be technically achievable and accepted by everybody.

\section{Acknowledgements}

The paper was prepared under the support of both the EU project THINKcreative (IST-2000-29478) and the Grant. No. MSMT 212300013 of the Ministry of Education, Youth and Sports of the Czech Republic.

\section{REFERENCES}

1. Camarinha-Matos, L.M., Afsarmanesh, H.: The Virtual Enterprise Concept. In: Infrastructures for Virtual Enterprises, Kluwer Academic Publ., 1999, pp.3-14

2. Camarinha-Matos, L.M., Afsarmanesh, H. (eds.): Interim Green Report on New Collaborative Forms and Their Needs, September 2002

3. Deen M. (ed): Holonic Manufacturing Systems. Springer Verlag, London, 2003

4. Mařík V., Pěchouček M., Štěpánková O.: Social Knowledge in Multi-Agent Systems, In: Multi-Agent Systems and Applications, LNAI 2086, Springer-Verlag, Heidelberg, 2001, pp.211-245

5. Novák, P., Rollo, M., Hodík, J., Vlček, T.: Communication Security in Multi-Agent Systems. In: Multi-Agent Systems and Applications III, LNAI No. 2691, Springer Verlag, Heidelberg, 2003

6. Pĕchouček, M., Norrie, D.: Knowledge Structures for Reflective Multi-Agent Systems: On Reasoning about Other Agents, registered as Report Number 538, Department of Mechanical and Manufacturing Engineering, University of Calgary, Alberta, Canada, 2000

7. Pěchouček, M., Mařík, V., Bárta, J.: A Knowledge-based Approach to Coalition Formation. IEEE Intelligent Systems, vol. 17, no.3, 2002, pp. 17-25

8. Shehory, O., Kraus S.: Methods for Task Allocation via Agent Coalition Formation. Artificial Intelligence Journal, vol. 101 (1-2), 1998, pp. 165-200

9. Sycara, K.: Multi-Agent Infrastructure, Agent Discovery, Middle Agents for Web Services and Interoperation. In: Multi-Agent Systems and Applications, LNAI No. 2068, Springer Verlag, Heidelberg, 2001, pp. 17-49

10. Tambe, M.: Towards Flexible Teamwork. Journal of Artificial Intelligence Research, vol. 7, 1997, pp. 83-124

11. Wooldridge M. : Reasoning about rational agents, The MIT Press, Cambridge, MA, 2000 